\title{
Le renouveau des politiques industrielles dans l'Europe de la Défense
}

David W. Versailles

\section{(2) OpenEdition \\ 12 Journals}

Édition électronique

URL : http://journals.openedition.org/ei/243

DOI : $10.4000 /$ ei. 243

ISSN : 2553-1891

Éditeur

Association Économie et Institutions

Édition imprimée

Date de publication : 30 octobre 2009

Pagination : 7-29

ISSN : 1775-2329

\section{Référence électronique}

David W. Versailles, « Le renouveau des politiques industrielles dans l'Europe de la Défense », Économie et institutions [En ligne], 12-13 | 2009, mis en ligne le 31 janvier 2013, consulté le 20 avril 2019. URL http://journals.openedition.org/ei/243 ; DOI : 10.4000/ei.243 


\title{
Présentation du numéro
}

\section{Le renouveau des politiques industrielles dans 1'Europe de la Défense}

\author{
David W Versailles
}

\begin{abstract}
Ce numéro spécial va tenter d'éclairer la situation des politiques industrielles en matière de Défense et de Sécurité, telles qu'elles ont évolué depuis le début des années 1990. Pendant la Guerre froide, toutes les politiques industrielles volontaristes en matière de Défense et Sécurité ont eu lieu par le biais d'agences particulières et de programmes spécifiques nationaux ou européens. $\mathrm{Au}$ titre de ces derniers, il est possible de citer les différents programmes d'avions de combat mis en œuvre en France sous l'égide de Dassault Aviation, ou encore le programme Eurofighter qui a permis à EADS d'acquérir des compétences particulières qui lui faisaient défaut. De même, pour la France, le développement de capacités en matière de détection sous-marine, de communication et de cryptologie a été associé aux ambitions des programmes de sousmarins nucléaires (lanceurs d'engins en particulier). Dans tous les pays, la mise en œuvre de capacités militaires particulières s'est traduite par l'introduction de plateformes spécifiques (avions, sousmarins et bâtiments de guerre, chars de combat par exemple) ou de systèmes de commandement et de communication. Ces programmes cristallisaient les efforts de tous les acteurs au sein de la base industrielle et technologique de Défense (Hartley \& Sandler, 1995 ; Mc Guire, 1995). La responsabilité au niveau des États était le plus souvent confiée à une agence ou à un service précis qui tirait aussi sa légitimité de l'aptitude à concrétiser une capacité militaire opérationnelle. Le CEA, en France, incarne parfaitement cette dynamique à travers la politique industrielle associée à l'énergie nucléaire, tant pour des missions civiles que militaires. Les compétences acquises dans le nucléaire conduisent la même agence à développer son rôle institutionnel en se voyant confier des responsabilités hors du nucléaire depuis de longues décennies.

A travers des études de cas concrètes, ce numéro spécial va tenter d'éclairer la situation actuelle des politiques industrielles en Europe. Il entend aussi contribuer à montrer l'intérêt de l'économie des institutions en la matière. Ces raisonnements peuvent être illustrés sur la base d'une référence aux missions de Défense et de Sécurité car celles-ci manifestent sous une forme radicale, sinon caricaturale, tous les points focaux du renouvellement du système
\end{abstract}


économique européen. Dans le nouveau contexte, la nature même de l'action publique évolue et introduit des besoins de coordination spécifique. L'existence conjointe de dynamiques de marché et de dynamiques hors marché a été identifiée depuis longtemps pour ces questions, mais l'appréhension du système productif requiert de nouvelles références pour prendre en compte les interactions entre niveaux européen, national et régional. La Défense était l'apanage des gouvernements nationaux, qui détenaient le pouvoir de marché à travers leur double situation de client unique (monopsoneur) et de donneur d'ordre public à travers les budgets nationaux. Même lorsque les États agissaient en tant que membres d'alliances internationales structurantes (par exemple l'OTAN), leurs décisions de politique industrielle en matière de Défense conservaient une forme de préséance qui n'existe plus aujourd'hui. Pendant les années de la Guerre froide, les caractéristiques sectorielles et nationales demeuraient assez faciles à cerner par la logique générale de la dissuasion nucléaire. Depuis le 11 septembre 2001, les États ont élargi de façon explicite le périmètre des décisions de Défense aux paramètres qui concourent à la fonction de Sécurité nationale, ce qui a fait exploser de façon définitive les frontières d'un secteur "Défense " qui était de toute façon de plus en plus "dual ", et augmenté dans le même temps la complexité de l'analyse des politiques publiques et de leurs conséquences en matière industrielle.

L'autonomie des niveaux nationaux a vécu depuis que les budgets des États ne permettent plus de renouveler leurs capacités militaires, de façon isolée, à la hauteur de menaces et des enjeux qui les concernent. Au-delà des aspects budgétaires et sectoriels qui positionnent les monopsoneurs face à leurs fournisseurs dans chaque pays, le système économique relatif aux fonctions de Défense et de Sécurité a été bouleversé par la mise en place d'une gouvernance politique et économique multi-niveaux où les prérogatives de chaque acteur ont été bouleversées. Les politiques économiques liées aux domaines de la Défense et de la Sécurité relèvent d'arrangements institutionnels complexes, liés au modèle intergouvernemental ou communautaire. La coordination des décisions publiques n'existe pas encore à tous les niveaux politiques et militaires, mais la restructuration des industries de Défense qui bat son plein depuis les années 1990 a déjà produit des acteurs supranationaux qui structurent le marché. L’asymétrie existe de façon patente car la décision politique "top-down" a perdu son caractère exclusif. Cette révolution dans la gouvernance des affaires industrielles se manifeste déjà par des dynamiques tout à fait structurantes au niveau du management de l'innovation, des logiques de privatisation de la connaissance, et des processus créatifs. Le contrôle et la diffusion des connaissances demeuraient 
simples quand les États géraient des logiques d'arsenal. Aujourd'hui, la nouvelle logique de l'économie fondée sur la connaissance prévaut, en matière de Défense et de Sécurité comme dans d'autres secteurs (Versailles, ed; 2005). Les logiques d'interfaces et de coopération entre l'Etat, la science et l'industrie ont changé. Cela conduit à s'intéresser à d'autres niveaux de l'interaction entre ces trois types d'acteurs, niveaux qui deviennent alors une cible privilégiée de la gouvernance des politiques publiques et de la gestion des processus concurrentiels.

Les articles de ce numéro spécial vont illustrer combien les concepts portés par les institutionnalistes permettent d'appréhender la complexité des politiques de Défense et de Sécurité. Ils appréhendent en particulier les effets contrastés consécutifs aux bouleversements survenus dans les vingt dernières années. De nouvelles formes d'intervention publique ont été installées progressivement. Le modèle qui prévalait pendant la Guerre froide était celui de la hiérarchie, qui régulait à la fois la coordination entre acteurs et toutes les questions liées aux pouvoirs de décision et aux incitations à contribuer aux programmes. Ce modèle a vécu. Du point de vue de l'analyse économique, les politiques économiques de Défense et de Sécurité sont intéressantes parce toutes les variables sont maintenant endogènes, y compris les formes des organisations, les structures de gouvernance, les outils de pilotage des arrangements internes qui répondent aux coûts de production et de transaction, et les modes de fonctionnement de la multi-gouvernance (degré de centralisation, degré de formalisation, etc.) qui se mettent en place. Toutes les organisations sont encastrées dans un environnement institutionnel qui se trouve lui-même en constante évolution, tout à la fois cause et conséquence des coûts de transaction qui pèsent sur chacun des acteurs de l'écosystème.

Les relations qui existent entre l'analyse économique et les questions de Défense sont anciennes et nombreuses. Elles ont été décrites depuis longtemps dans le Handbook of Defence economics publié en 1995 (Hartley et Sander, eds ; 1995). Elles concernent des outils analytiques et des objets, en relation avec une analyse normative visant à l'élaboration et à la mise en place de politiques publiques (McGuire, 1995 : 39). Les outils actuels ont permis de pousser plus loin l'étude de la dynamique d'interaction, ou encore la compréhension des mécanismes de R\&D. Les travaux menés soulignent que tous les acteurs se projettent dans des raisonnements qui portent sur de très longues périodes de temps, où la gestion des actifs incorporels occupe une place majeure (Versailles, ed ; 2005). Les activités concernées sont toutes hautement intensives en connaissance; les systèmes de Défense sont le plus souvent reliés à des technologies émergentes et supposent des innovations d'usage au moment de leur développement. Dans le même temps, tous les 
systèmes sont mis en œuvre dans la longue durée, ce qui impose de préserver les compétences associées à toutes les générations de technologie pendant plusieurs décennies (ibid: 87). La politique industrielle de Défense est structurée par cette tension permanente entre besoin de créativité et gestion des legacy systems ${ }^{1}$. Tous les acteurs du système conservent ces contraintes en ligne de mire. Ils regardent en même temps vers l'avenir et vers le passé. Les effets de réseau et les mécanismes de transfert (ou de conversion) des actifs incorporels régulent les comportements (Bellais \& Guichard, 2006). C'est ici que l'analyse trouve les critères de rationalité qui expliquent les comportements et les choix de chaque acteur, qu'il soit industriel, chercheur ou encore représentant de l'État dans sa double fonction de donneur d'ordre (qui décide de la politique de Défense et paie les programmes à partir du budget de l'État) et d'usager (le soldat qui remplit sa mission et transmet son expérience opérationnelle et ses retour d'expérience). La conjonction de ces perspectives explique l'alternance entre exclusion et obéissance aux lois du marché.

Ce numéro thématique se propose d'illustrer quelques pistes de recherche en matière d'analyse économique des institutions, sans prendre parti au sein de la diversité des institutionnalismes ni tenter de faire système au niveau de l'analyse des questions de Défense. Il poursuit également la démarche de la Defense economics (par opposition à Economics of Defense, cf Schmidt 1987), où la Défense est appréhendée avec les outils traditionnels de l'analyse économique. Les travaux présentés dans ce numéro spécial font état d'investigations développées dans des contextes divers, qui se fondent toutes sur des recherches de terrain. Certaines avaient été développées pour éclairer les décisions publiques ou procurer un retour d'expérience sur des outils de politique publique. Sous une forme ou une autre, tous les programmes d'armement et toutes les politiques publiques de Défense peuvent être mobilisés pour les réflexions autour des thèses institutionnalistes, à la condition que les informations deviennent disponibles pour le grand public. Dans de nombreux cas, la protection des données relatives à la Défense rend difficile la référence à des technologies ou à des matériels tant que leur caractère critique demeure, ou tant que les matériels sont en service. Parfois, les données accessibles au grand public (ou au chercheur) sont partielles et, donc, peuvent induire des

1 On désigne sous ce nom les systèmes technologiques de générations diverses et variées qui interagissent avec les systèmes les plus modernes au sein des forces armées. On parle parfois de systèmes "hérités ". Les matériels subissent en permanence des mises à jour et des adaptations pendant leur vie opérationnelle, qui est longue de plusieurs décennies dans la Défense. Sont concernés à la fois les logiciels ou les sous-systèmes technologiques. Le problème consiste à gérer l'hétérogénéité des spécifications techniques aux niveaux opérationnels et industriels. 
interprétations erronées ou abusives parce que le chercheur prend seulement en compte ce qui se voit, et laisse de côté ce qui ne lui est pas donné à voir. C'est ici que l'ambigüité du terme institutionnel doit être levée. Quand les aspects institutionnels renvoient aux prérogatives des acteurs, les faits à analyser sont assez facilement disponibles car ils renvoient à des éléments publics. Calibrer la nature réelle de ces activités et la réalité des échanges suppose de pouvoir entrer dans des niveaux de détail qui impliquent des méthodologies différentes. Les articles de ce numéro spécial se sont appuyés sur des investigations commandités par la Défense au cours de la dernière décennie, ou alors ont été menés quand certains des auteurs faisaient eux-mêmes partie de l'institution de Défense et y étaient actifs en tant que chercheurs. Ils ont ainsi eu accès à des données de première main; ils ont pu directement confronter théories et réalité concrète. De plus en plus de travaux de ce type sont possibles sur les questions de Défense.

Cette introduction va revenir dans un premier temps sur différents niveaux pertinents pour développer l'analyse des politiques industrielles de Défense, avant de positionner ces débats face à la diversité des institutionnalismes. Elle présente ensuite quelques éléments de dynamique qui situent les politiques publiques dans le nouveau système avant d'exposer, avec quelques points saillants tirés de chaque article de ce numéro, les outils principaux qui ouvrent de nouvelles perspectives aux politiques industrielles en matière de Défense.

\section{Politiques Industrielles et Defense}

Traditionnellement, les politiques industrielles en matière de Défense couvraient les sujets liés à l'acquisition des programmes d'armement et l'" efficacité " de la dépense budgétaire. Ces mécanismes reposent sur quatre piliers (Hartley, 1995), qui sont appréhendés par les outils traditionnels de l'organisation industrielle :

- la standardisation des matériels au sein des alliances militaires (par exemple au sein de l'OTAN) ;

- la mise en œuvre d'effets de série au niveau des programmes d'armement, dans le cadre de production sous licence, d'exportations et de coopérations militaires avec des partenaires stratégiques ;

- l'analyse des retours industriels et des "offsets ";

- la possibilité de dégager des ressources budgétaires à partir d'une amélioration du fonctionnement des forces armées (ce que Hartley désigne comme la fonction de production militaire) ou de la conversion d'infrastructures militaires inutiles, ressources qui seraient "injectées " dans la base

11 Economie et Institutions - n²12\&13 -2008-2009 
industrielle et technologique de Défense au niveau de la R\&D ou de la production des systèmes d'armes.

Ces questions ne font pas référence aux processus euxmêmes, mais plutôt aux inputs de la Défense et au partage de la charge budgétaire ("burden sharing" dans la terminologie de l'OTAN). Ces démarches peuvent être interprétées comme des politiques sectorielles qui visent à encadrer les mécanismes de marché. Au-delà des questions budgétaires, elles permettent aux États de " manager la concurrence " dans le cadre de l'attribution des marchés publics, afin de sélectionner le meilleur fournisseur tout en permettant aux "perdants" de ne pas sortir du marché. Les procédures mises en œuvre visent à protéger les marchés nationaux de la concurrence étrangère, ou encore à passer outre aux considérations économiques dans l'attribution des marchés. De nombreuses mesures pro-actives ont ainsi été mises en place, qui ont d'abord ciblé la préservation des compétences nationales sous une forme implicite, avant de devenir le point majeur de la politique industrielle. C'est par exemple le cas au Royaume-Uni où les critères de maintien des capacités industrielles et technologiques à long terme font maintenant partie des critères fondamentaux retenus pour les acquisitions d'armement (House of Commons - Defence Committee, 2003 ; MoD Defense Industrial Strategy, 2005). La même logique prévaut en France avec les procédures de marchés négociés. Les États-Unis se singularisent, quant à eux, par une forme de dichotomie entre un discours libéral et une réalité concrète marquée par une intervention récurrente de l'État fédéral dans la structuration des marchés de Défense.

Tous les pays ont adopté un discours lié à la préservation de leurs capacités militaires. L'histoire des XIXème et XXème siècles européens regorge d'exemples de renversement d'alliances et de confrontations plus ou moins chaudes qui expliquent, en même temps, pourquoi ces pays ont mené des politiques industrielles autarciques qui répondaient à la nécessité de sécuriser les approvisionnements dans la durée. La logique de confrontation (armée) et de course aux armements s'est traduite par des duplications industrielles et des redondances de compétences dont a hérité la Guerre froide. En Europe en particulier, cette situation n'a pas pu durer et a nécessité des restructurations progressives. En France, la naissance de la Vème République coïncide avec une politique visant à obtenir l'autonomie d'approvisionnement en matière technologique pour le développement de tous les systèmes d'armes. La politique d'armement est associée à la volonté d'être une puissance reconnue à l'échelle internationale, refusant la bipolarité et voulant préserver la maîtrise et l'autonomie de décision. Aux États-Unis, la politique d'armement a toujours été guidée par la 
recherche et le maintien de la supériorité technologique des forces armées (Bishak, 1999). Gérer ce principe à l'échelle d'un continent suppose aussi la prise en compte de contraintes particulières, car il est évident que la base industrielles et technologique de Défense américaine se trouve pour l'essentiel sur le contient nord-américain. Des dispositions visant à exiger des sous-traitants des garanties dans l'approvisionnement de l'industrie de Défense se trouvent donc légitimes au regard de la logique industrielle. Il demeure toutefois surprenant que cette réalité puisse s'accommoder de situations particulières avec des alliés précis (Japon, Israël) quand de nombreuses entreprises européennes, par exemple, se voient opposer cet argument même lorsqu'elles s'allient avec des entreprises locales pour produire sur le continent américain. Faire la part des choses entre réalité opérationnelle de la sécurité des approvisionnements, protectionnisme, et gestion de la chaîne de valeur devient donc difficile.

La gestion de l'autonomie d'approvisionnements suppose donc de gérer des complémentarités et des choix stratégiques de localisation de compétences. Dans le contexte géostratégique particulier de la Guerre froide, structuré par deux pôles majeurs, les alliances militaires volontaires (comme l'OTAN) ont permis d'introduire une forme de rationalisation des capacités industrielles qui montre toute sa limite, par exemple, avec la fin du Pacte de Varsovie. Au moment où les anciens pays satellites rejoignent le giron de l'OTAN sous des formes diverses, la base industrielle et technologique de l'ancien pacte de Varsovie se trouve amputée de nombreuses compétences. Il en va de même entre les États-Unis et leurs alliés principaux. Le Royaume-Uni a connu ce débat dès 1964. Le gouvernement britannique avait alors reconnu qu'il n'avait plus les moyens, pour des raisons budgétaires, de préserver un outil de production pour l'ensemble des moyens militaires. Le pays est alors entré dans une logique de partenariat privilégié et de partenaire politique crédible acceptant une certaine dépendance technologique vis-à-vis des États-Unis (Freedman, 1999). L'adoption de la technologie américaine pour les sous-marins nucléaires de la série Trident en constitue une illustration emblématique. La contrepartie de cette décision réside dans le contrôle qu'opèrent les Américains sur les décisions de transfert technologique associées à ces domaines mais, surtout, sur les conditions d'emploi opérationnel par la Marine britannique.

A l'échelle de l'Europe, ces questions se posent sous une forme différente. Les décisions en matière de Défense renvoient à des modes de décision qui relèvent du Deuxième Pilier de l'Union européenne. Les modes de répartition de rôles et de décision sont spécifiques, associés à des structures institutionnelles comme l'OCCAr (Organisme commun de coopération en matière 
d'armements) et l'Agence Européenne de Défense (AED). La mise en place du millefeuille institutionnel européen a bouleversé tous les échelons qui existaient auparavant au niveau national. De nouvelles questions se posent, qui renvoient directement à une logique de localisation géographique des activités industrielles. Dans le cadre de la coopération européenne, elles prennent une connotation particulière car elles sont associées à deux processus parallèles. Le premier concerne la coopération entre Etats, et en particulier la façon dont l'acquisition des matériels et l'emploi des forces sont coordonnés à l'échelle multinationale. Le second est relatif à la restructuration des industries de Défense dans le cadre européen (avec la création EADS, ou la restructuration de BAe Systems et Thalès par exemple). Après deux décennies d'évolution environ, force est de constater que ces vitesses d'ajustement ne sont pas tout à fait coordonnées. La décision politique ne conduit pas à des choix de spécialisation industrielle et il demeure impossible de se concentrer sur les acteurs qui présentent les meilleurs avantages comparatifs. L'industrie ne pouvant attendre sine die des décisions politiques coordonnées, elle s'est restructurée selon des stratégies propres et a produit des "champions " multinationaux dont les périmètres ne sont pas tous cohérents avec la construction européenne (ainsi de l'alliance stratégique entre les industries italiennes et américaines). Cette situation induit encore des duplications inutiles et l'impossibilité de générer toutes les économies d'échelle possibles. S'il était possible de prendre des décisions politiques claires sur la Défense européenne, les restructurations industrielles pourraient aller plus loin dans la recherche d'avantages comparatifs, les coûts de coordination et de transactions pourraient encore être diminués, et l'efficacité de la dépense publique de Défense pourrait s'en trouver améliorée à l'échelle européenne.

\section{REGARDS DES INSTITUTIONNALISMES SUR L'ANALYSE ECONOMIQUE DE LA DEFENSE}

Les investigations de ce numéro spécial s'inscrivent résolument dans la nouvelle économie institutionnaliste qui inclut non seulement l'école des coûts de transaction (Coase, North, Williamson), mais aussi celle des théoriciens des droits de propriété. Les travaux désignés par Aoki comme l'analyse comparative institutionnelle sont également pris en compte ici. De Veblen et Commons à Williamson, North, Aoki et Hodgson, en passant par Carl Menger et par certains membre de l'Ecole autrichienne, les thèses institutionnalistes comparent la nature des institutions, les institutions paradigmatiques, les références formelles et/ou informelles, le statut des organisations par rapport aux institutions et, enfin, le moteur de l'évolution. Toutes ces références identifiées

14 Economie et Institutions $-\mathrm{n}^{\circ} 12 \& 13-2008-2009$ 
par Chavance $(2007: 104-105)$ pour souligner la diversité et l'unité des institutionnalismes se montrent pertinentes pour analyser la Défense, car elles permettent d'aborder un point fondamental sous des formes variées. Pour aborder ce terrain concret dans toute sa réalité, l'analyse économique doit étudier les aspects institutionnels comme des variables endogènes à son champ. Un corolaire de ce constat existe au niveau de la dimension historique qui touche à la relation logique entre la configuration institutionnelle et le système économique qui l'entoure. $\mathrm{Vu}$ la dynamique particulière de la construction des compétences et les contraintes particulières qui concernent la gestion des programmes de Défense, la dimension évolutive est incontournable. La dynamique du changement doit être appréciée dans toutes ses nuances, pour tous les acteurs, dans une logique de co-évolution. Au niveau micro-économique (par exemple en termes de management de la technologie ou de gestion de l'innovation), l'intérêt pour les processus prime sur les approches centrées sur l'équilibre car les acteurs raisonnent dans le temps long. Dans une perspective plus macro-économique, le constat est le même à cause de la liaison directe entre les politiques publiques et les questions budgétaires, qu'il convient toujours de raisonner par rapport à un cadre international. Ainsi qu'on le retrouve régulièrement dans les travaux de la famille institutionnaliste, c'est le différentiel de changement (pour reprendre les mots de Chavance) qui présente un intérêt parce qu'il permet de souligner les temporalités relatives des processus économiques. Ce différentiel s'applique à la fois aux institutions qui fondent l'existence du marché, et à celles qui permettent son fonctionnement (pour reprendre les termes de Coriat et Weinstein, 2002 ; 2004).

Aoki (2000: 161) propose de distinguer quatre grands groupes d'institutions, qui éclairent toutes sous un jour différent les questions relatives à la Défense : le marché en tant que tel (et la monnaie) ; l'Etat et les appareils d'Etat; les structures de statut privé ; les normes sociales et les croyances partagées. Chacune des quatre catégories éclaire de façon pertinente les sujets relatifs à la Défense. Cette typologie permet aussi de souligner combien les analyses qui empruntent aux thèses institutionnalistes apportent une véritable valeur ajoutée à l'étude des organisations de Défense.

Sur le marché tout d'abord: Toutes les théories relatives à l'analyse économique de la Défense explicitent que ces marchés sont administrés, et qu'ils doivent le demeurer pour satisfaire efficacement la mission de Défense. La question n'est pas de savoir si les marchés relatifs à la Défense peuvent se coordonner sur la seule base des signaux procurés par les prix, car elle ne suffit pas à épuiser le problème. Les biens échangés et les enjeux sont bien trop complexes pour que les prix suffisent à résumer les interactions entre agents et pour que la rationalité des agents s'accommode d'une 
coordination par les prix. La politique de Défense remplit des missions qui dépassent largement la problématique du marché, ce qui suppose que les agents introduisent des réponses économiquement viables à des questions non-économiques. C'est ici que la question traditionnelle de la confrontation entre offre et demande (qui se matérialise par l'existence d'un monopsone) se trouve complétée par une dimension institutionnelle : elle repose à la fois sur la hiérarchie des acteurs (Williamson), structurée par tantôt l'Etat, les usagers, ou l'industrie, et aussi sur la confiance qui relie ces acteurs à travers un mécanisme d'échange (Carl Menger). Le côté demande tient à sa disposition un ensemble de règles budgétaires et réglementaires grâce auxquels son pouvoir de marché est complété, préservé ou conforté. Les articles de ce numéro vont illustrer toute cette complexité. Même dans les pays les plus dotés au niveau budgétaire (ce qui accroît fortement le pouvoir de marché du client de référence aux niveaux national et international), la relation de marché échappe largement à une simple asymétrie de pouvoirs économiques entre le client (l'Etat) et ses fournisseurs (l'industrie en particulier). La véritable question est celle de "l'architecture du marché ", comme la désignent Coriat et Weinstein (2004: 53), à partir des différents groupes d'agents et des modes de relation entre ces groupes. C'est cette architecture très enchevêtrée [nested] qui permet de caractériser la "négociation " entre l'industrie et le(s) client(s).

Sur l'appareil d'Etat ensuite: Le pouvoir du monopsoneur dépend du budget public disponible pour la Défense, mais aussi d'un grand nombre de variables qui nuancent sa capacité d'intervention. Depuis très longtemps, les auteurs de la science politique sont venus compléter les approches typiquement économiques pour leur donner un contenu plus dense au niveau de la nature des institutions et, aussi, pour donner sens à des institutions informelles (North) qui viennent compléter les aspects les plus formels. Pour bien identifier les causalités, il ne faut pas oublier de regarder dans la partie immergée d'un iceberg qui suppose une connaissance interne détaillée (" emic" ou "insider " pour reprendre les termes de l'anthropologue Marvin Harris ; Headland et al, 1990) de l'institution et des interactions qui la relient à l'ensemble des acteurs. Les représentants de l'industrie, de la recherche et de l'administration font partie d'un système de règles sociales encastrées, qui structurent toutes les relations et codifient les rapports fondamentaux. Les règles du jeu sont plus ou moins figées selon les pays et les époques. Les connaitre représente une véritable barrière à l'entrée dans ces réseaux d'interaction, barrière qui joue largement de l'existence de règles de confidentialité même si cette référence relève bien plus souvent du cosmétique que de la réalité. Ces démarches tendent à augmenter les coûts d'entrée des "outsiders ", 
à travers des coûts de transaction et des coûts d'information très élevés (Coase). L'appareil d'Etat n'est jamais un acteur homogène, il est constitué d'une pluralité d'acteurs qui caractérise chaque Etat, où chacun joue sa propre partition et poursuit des objectifs et des stratégies spécifiques. Aujourd'hui, les chercheurs qui travaillent sur les politiques publiques de Défense incorporent automatiquement une analyse des systèmes de règles sociales encastrées dans les interactions. Ils ne peuvent pas analyser les questions relations au management de la technologie (ou de l'innovation) sans introduire, par exemple, une analyse micro-économique des relations de dépendance au sentier (North) et de "lock-in" en termes de compétences (individuelles ou collectives). Ces questions viennent tout de suite à l'esprit lorsqu'il est question de structures de statut privé, mais l'analyse des politiques publiques en matière d'innovation et de recherche montre que leur efficacité dépend directement des compétences des acteurs publics et des modes de gouvernance que ces compétences permettent.

Sur les structures de statut privé : L'industrie de Défense a longtemps vécu sous un statut public à travers les logiques d'arsenal. Aujourd'hui, sous le coup des restructurations et des évolutions technologiques, les industries à statut public ont presque toutes disparu. Cela signifie que les acteurs publics doivent prendre en compte des stratégies privées et entrent dans des logiques de négociation qui ne portent plus seulement sur les questions de coût ou de rentabilisation des efforts de recherche à travers la production en série des matériels. Cette situation conduit à deux conséquences. La première renvoie à la compatibilité des stratégies entre les acteurs publics et privés, et ainsi à la possibilité d'installer une relation d'agence. Tous les risques identifiés à ce niveau doivent être analysés de façon concrète: asymétrie de l'information, alea moral, antisélection (et émergence de passagers clandestins). La deuxième conséquence renvoie à la nécessité d'installer des modalités de gouvernance qui partagent la décision entre les structures privées et publiques, ce qui intervient à la fois au niveau de programmes particuliers (par exemple un avion de combat spécifique), d'un programme de R\&D (par exemple la furtivité des matériels, ou les systèmes de communication en temps réel), ou de la fusion de ces deux démarches en un seul projet à la complexité accrue (par exemple le programme d'avion de chasse JSF aux Etats-Unis, qui réunit les exemples précédents). In fine, c'est la question de la nature des politiques publiques et des leviers de l'intervention publique qui est posée à travers l'émergence des nouveaux rôles des structures de statut privé, et du partage de la décision publique qui en découle.

De façon plus générale, les chercheurs qui travaillent sur la Défense considèrent tous que les organisations sont à la fois des institutions et des joueurs (Aoki) qui interviennent à tous les niveaux du système 
économique pertinent pour les décisions publiques et dans l'organisation industrielles reliée aux programmes de Défense.

Sur les normes sociales et les croyances partagées, enfin: Par nature, les institutions relatives à la Défense sont ancrées dans des modèles de gouvernance de transactions (Williamson). Les interactions se réfèrent en permanence à un système de règles du jeu formelles et informelles (North), le revalident à chaque instant et l'ajustent pour mieux lui permettre de structurer l'avenir. Cet environnement s'apparente à un système auto-entretenu de croyances sur la façon dont le jeu institutionnel est joué (Aoki), qui en vient à constituer un véritable paradigme cognitif qui structure les comportements et les interactions encore longtemps après qu'il a cessé de reposer sur la réalité qui lui a donné naissance. Dans tous les pays où les politiques publiques de Défense jouent ou ont joué un rôle majeur, ce genre de phénomène existe. Les modalités de survie de ces schémas sont très diverses. Le plus important renvoie aux mécanismes qui structurent les hiérarchies formelles ou informelles entre acteurs, et qui fondent la légitimité de chaque famille d'acteurs au niveau national. Dans les réseaux liés à la Défense, chaque pays manifeste sur cette base une forme de codification des rapports " sociaux " qui est propre à son système de formation et à la mobilité des acteurs entre l'industrie, l'administration en charge de l'acquisition, les usagers (militaires) et les organismes de R\&D. Ces éléments font système (Hodgson), ce qui explique que l'on compare souvent la France, les Etats-Unis et le Royaume-Uni car ce sont trois pays de l'OTAN pour lesquels la place de la Défense dans les politiques publiques reste essentielle. On utilise ces caractéristiques pour singulariser les systèmes nationaux, les comparer et analyser des domaines précis, comme l'innovation. L'originalité du système de formation peut être illustrée par exemple avec le système des grandes écoles françaises qui fabrique en même temps les élites de l'industrie, de la recherche, de l'administration et des usagers. Les mécanismes de mobilité des individus singularisent l'imbrication des relations pour chaque pays : on peut représenter en même temps l'administration et l'industrie en Grande-Bretagne, ou enchaîner des postes chez tous les contributeurs au sytème de Défense dans une carrière chez les Américains. En France, le système qui prévalait jusqu'aux années 1990 (et qui était fort similaire à ce qui existait aux Etats-Unis en matière de résultats) a évolué ; les agents publics sont maintenant sur des parcours professionnels plus typés qui ne permettent plus les mêmes mécanismes de transmission de connaissances et occasionnent des coûts de transaction très élevés et l'émergence de coûts irrécouvrables. Normes sociales et croyances ont un impact très important sur les mécanismes de coordination et sur la possibilité de faire émerger des anticipations (ou des stratégies) mutuellement compatibles. 
La dimension évolutionniste et historique est indispensable pour interpréter les questions de Défense, qui s'inscrivent dans le temps long. Les orientations sont fondées sur des mécanismes endogènes de pouvoir qui dépendent des règles, et dans lesquels les acteurs sont tous régulés in fine par les contraintes budgétaires. Des économies sont toujours possibles en optimisant les structures de coûts (Williamson) (transaction, information), et les mécanismes de conversion de connaissance. De façon générale la dépendance au sentier affecte largement toutes les activités de Défense. Tous ces mécanismes seront illustrés dans les articles de ce numéro spécial.

\section{LeS NIVEAUX D'ANALYSE DE LA DEFenSe}

L'analyse économique de la Défense a été souvent influencée par d'autres disciplines, comme la science politique. Ainsi, pendant la Guerre froide, les relations entre la Défense, la recherche publique et les entreprises était le plus souvent associées à la notion de complexe militaro-industriel. Le terme marquait non seulement une relation étroite entre les acteurs contribuant aux programmes d'armement, mais surtout une imbrication entre les activités d'un nombre limité d'acteurs publics et privés (Lieberson, 1972). Il reflétait l'existence d'un réseau d'acteur hermétique au reste du système économique. Les auteurs de la science politique américaine qui ont forgé le concept (Schwartz, 1990) faisaient référence à une collusion d'intérêts visant à des augmentations récurrentes et peu modérées de la dépense publique de Défense, et à la promotion de décisions politiques favorables à quelques acteurs industriels ciblés sur la base du lobbying de quelques parlementaires très impliqués. C'est pour cette raison que le Président américain Eisenhower a utilisé le terme dans son discours de fin de mandat en 1961. Dans son acception sociologique, le concept est totalement compatible avec une interprétation holiste. Les intérêts stratégiques des acteurs qui le composent conduisent à des dépenses sous-optimales à cause même de la structure d'agence mise en place, et le complexe militaroindustriel se trouve alors représenter un exemple du paradoxe " agent-principal ". Aller plus loin en analyse économique suppose d'entrer dans le détail de ce que les Américains désignaient alors comme " the iron triangle ", reprenant les trois pôles du politique, de l'industrie et des forces armées (ou des militaires selon les auteurs) et de détailler les différents acteurs qui se partagent les responsabilités au niveau des programmes relatifs à la Défense.

Depuis quelques années, les économistes (Dunne, 1995) caractérisent la notion de complexe militaro-industriel par la prégnance d'une relation de dépendance à la commande publique, dans un contexte où règnent les règles de confidentialité liées à la protection des programmes de Défense. Les acteurs pertinents pour 
développer l'analyse sont alors situés au niveau de la décision sur les montants et l'affectation des budgets publics, ou alors au niveau de leur emploi. Dans une perspective plus micro-économique, les acteurs seront alors circonscrits dans une typologie où l'on retrouve les acteurs étatiques (dont le militaire), l'industrie et, aussi, les acteurs de la R\&D. C'est sur l'acteur étatique que porte potentiellement la plus grande imprécision lorsqu'on parle simplement de "Défense ", ambigüités qui doivent être levées pour bien identifier la dynamique des choix publics. La multiplicité des rôles associés l'État se retrouve à travers trois activités distinctes : les forces armées qui regroupent les usagers, les agences et les gouvernements qui préparent les processus d'acquisition, et aussi les parlementaires qui valident in fine les budgets. Ce souci de précision permet, au passage, de gommer les spécificités de l'organisation politique américaine au sein de l'analyse économique des choix publics, et de prendre en compte les relations entre gouvernements et parlements en Europe. Le paysage des acteurs de la Défense étant alors clarifié, il reste seulement à se référer à cette typologie à chaque fois que le niveau de l'analyse doit être précisé.

Le niveau macro présente des caractéristiques faciles à identifier, et pertinentes tant que l'analyse prend sa source dans des données budgétaires et des considérations d'ensemble. La Défense dispose en effet des cinq critères d'un acteur collectif définis par Scharpf (1997) et Le Galès (2003). Elle est constituée d'un système de décisions collectives entre les différents services qui la composent. Elle définit et représente des intérêts particuliers dans un ensemble plus vaste. Elle dispose de mécanismes d'intégration et d'arbitrage des conflits en interne ainsi que d'une représentation externe. Enfin, elle est capable d'innovation dans la définition de sa stratégie. Partagées par tous les acteurs, les références recoupent alors trois dimensions : réglementaire, normative et cognitive. D'un point de vue micro, les institutions peuvent être perçues comme des structures qui émergent de l'interaction entre acteurs dans la vie courante, et acquièrent progressivement un caractère interpersonnel en s'affranchissant des acteurs qui les ont créées. Les dimensions réglementaire, normative et cognitive peuvent alors être considérées comme des contraintes construites par les individus qui orientent et coordonnent leurs actions dans la vie courante (North, 1990). L'imbrication entre les niveaux individuels et collectifs est très structurée, ce qui permet d'articuler sans difficulté les niveaux micro et macro tout en demeurant cohérent avec l'individualisme méthodologique (Agassi, 1975 ; Boland, 2003). Pour les chercheurs qui veulent se concentrer sur le niveau micro, et étudier justement les trois dimensions citées, les informations nécessaires sont disponibles: l'action des acteurs fait sens par rapport à leur environnement, car chacun d'eux saisit des opportunités et gère ses 
contraintes propres au sein d'un ensemble plus vaste (Crozier et Friedberg, 1977). Les aspects institutionnels vont alors faire partie d'un ensemble de schémas, catégories et modèles cognitifs internalisés dans les comportements et dans les raisonnements individuels. A travers cette relation, le contexte institutionnel va pénétrer les organisations en définissant les champs du possible de l'action collective, par le biais des croyances et des idées des individus qui la composent. Les représentations, les idées et les valeurs collectives représentent alors une dimension centrale de l'analyse des politiques publiques. Si l'on pousse plus loin l'analyse en terme d'analyse économique, on entre alors dans les démarches liées aux dynamiques de création et de coévolution entre connaissances individuelles et collectives qui sont reliées aux approches en termes d'actifs de connaissance qui se développent, par exemple, en économie de l'innovation (cf Versailles, ed ; 2005 ; Amin et Cohendet, 2004 ; Mérindol \& Versailles, 2010).

Les travaux présentés dans ce numéro spécial se situent dans ce continuum d'analyse entre micro et macro. Les objets privilégiés par les auteurs concernent les dimensions réglementaires, normatives et cognitives, mais les analyses réalisées empruntent à la fois aux deux niveaux. Les réseaux d'acteurs représentent une matrice globale qui permet d'envisager une variété d'interactions et d'échanges entre individus, groupes et organisations dans le cadre d'un domaine d'activité particulier (et contextualisé). Au titre des groupes et des organisations, les développements prendront en compte des sous-ensembles de l'appareil d'État ou de l'industrie, au niveau national ou international. L'organisation se différencie ici des réseaux et des communautés d'acteurs par l'existence de mécanismes hiérarchiques, de structures formelles et stables dans le temps. Pour reprendre la terminologie de Coase $(1995$; 2000), la structure complexe de la Défense est de nature "réticulaire " (interrelated). Le coût de la coordination et le niveau des coûts de transaction sont affectés par les échanges entre les acteurs du système de Défense. Les coûts supportés par chaque acteur affectent tous les autres acteurs, au point de faire système car chacun dépend des coûts de coordination et de transaction introduits par les autres. Avec à la Défense, le chercheur se trouve confronté à un système complexe de relations d'interdépendance qui justifie le recours à la démarche "néo-institutionnelle ".

La nécessité d'alterner entre niveaux micro et macro est cohérente avec les différents rôles joués par la Défense dans les réseaux d'acteurs. Du point de vue de l'organisation industrielle, ces différents aspects demeurent difficiles à appréhender dans le cadre d'une démarche macro ou holiste, et c'est la raison pour laquelle ils sont commentés dans les articles de ce numéro spécial au regard de la typologie des acteurs introduite dans cette section. Au niveau des

21 Economie et Institutions - n $12 \& 13-2008-2009$ 
rôles joués par la Défense, cela se traduit par un double comportement de maitre d'ouvrage et d'usage innovant (lead user; Mérindol, 2009) impliqué dans le processus d'émergence et de validation de l'innovation. Ces rôles ont existé depuis toujours, mais sous des formes diverses. L'intervention de l'Etat dans le processus de conception et de production des systèmes de Défense s'est d'abord traduite par l'exercice d'une responsabilité directe en tant que maître d'ouvrage. Quand l'État agit comme le propriétaire final spécifiant les attentes, contrôle les programmes de $R \& D$ (de la recherche amont la plus générique jusqu'aux développements les plus finalisés) et la réalisation de l'architecture des systèmes et des sous-systèmes, il se comporte comme tel (Cohendet et Lebeau, 1987). Ce rôle est assez simple à définir en référence à un cumul entre le rôle d'intégrateur système (Prencipe et al., eds ; 2005 ; Hobday, 1998) et à un droit de propriété sur les systèmes technologiques et les actifs incorporels qui leur sont relatifs (droits de propriété intellectuelle). Mais cela ne suffit pas à caractériser les comportements de la Défense. Elle intervient aussi comme un usager particulier de la technologie impliqué dans le processus de créativité à cause de son expérience concrète de la finalité opérationnelle des systèmes (von Hippel, 1986 ; 2005). Individus et organisations peuvent détourner l'usage initial de la technologie, ou encore l'étendre à d'autres fonctions. Bien qu'il ne se situe pas sur même plan que la responsabilité de maître d'ouvrage, ce rôle aussi traduit une certaine capacité à innover en interne et à co-concevoir les systèmes avec les fournisseurs. La variété des processus d'innovation conduit à envisager le coexistence de ces statuts, qui traduisent des formes différentes d'intervention de la Défense dans les projets technologiques. Les statuts de maitre d'ouvrage ou de lead user nécessitent l'existence d'une capacité des organisations de Défense à fournir de manière transparente des axes d'intervention et à jouer un rôle adapté dans l'ensemble des dispositifs d'action publique. Ces modèles se révèlent essentiels pour comprendre les mécanismes de production et de diffusion des connaissances.

En matière de Défense, la nécessité d'une analyse multiniveaux seule permet d'appréhender sous une forme "compréhensive" la complexité des relations entre les acteurs économiques. Les individus, les groupes et les organisations font évoluer les mécanismes; ils s'adaptent eux-mêmes et évoluent en fonction des identités, des modes d'organisation et des formes d'interaction qui résultent de leurs propres activités. Toutes ces variables peuvent être analysées de façon endogène. Les thèses institutionnalistes procurent précisément les outils qui permettent de se confronter à ces enjeux analytiques. 


\section{DE NOUVEAUX OUTILS DE POLITIQUE INDUSTRIELLE EN MATIERE DE DEFENSE}

Les articles de ce numéro proposent six éclairages sur les politiques industrielles en matière de Défense. Ils ne prétendent pas épuiser la thématique, ni proposer un système d'analyse exhaustif.

Nathalie Lazaric, Valérie Mérindol et Sylvie Rochhia analysent l'évolution de la position de la Délégation générale pour l'armement (DGA) dans la chaîne de valeur des acteurs de la Défense à la suite des différentes réformes qui ont affecté cette structure depuis la fin des années 1990, jusqu'à la faire redevenir une Direction avec la dernière réforme du ministère. A travers la DGA, c'est le rôle du ministère français de la Défense qui évolue. Partant d'une fonction de maître d'ouvrage et d'intégrateur système, à l'influence encore renforcée par son pouvoir de marché (client), la situation a évolué progressivement à travers une succession de réformes. Les auteurs insistent sur l'impact de ces bouleversements sur les compétences détenues en interne, à la fois au sein du ministère français de la Défense et au sein de la DGA. L'analyse du changement institutionnel permet d'apprécier la dynamique de la division du travail comme une conséquence de la division des connaissances. Les auteurs montrent combien la nature même de l'intervention publique est affectée à la fois par l'évolution des compétences détenues par les agents de l'Etat, et par la répartition de ces compétences au sein de l'institution de Défense.

Victor Dos Santos Paulino développe son analyse sur le secteur spatial, et se concentre sur le lien entre management de la technologie, risque et inertie. Il montre l'évolution des coûts de transaction et d'information qui découle de l'accroissement de la maturité des technologies, et qui se manifeste par la transition de l'exploration de nouvelles technologies vers l'exploitation de solutions matures. Le secteur spatial a été longtemps associé à des missions régaliennes qui touchaient directement les missions de Défense et de Sécurité. L'arrivée de nouveaux acteurs liés aux communications civiles dans le secteur spatial transforme les relations de marché. L'environnement institutionnel évolue et change tous les contours du secteur ce qui implique que le changement technologique n'est pas favorisé. Les critères de rigueur qui s'appliquent à la sélection des technologies se durcissent et induisent des comportements d'aversion au risque. Que cela concerne les marchés matures liés à l'exploitation de technologies pour des marchés grand public n'est pas surprenant. Le problème souligné par l'auteur renvoie à la généralisation de ces comportements pour tous les acteurs du domaine, qu'ils touchent les missions grand public ou la Défense et les missions régaliennes. 
Thomas Le Texier et David W Versailles analysent l'impact de l'introduction des mécanismes d'innovation "ouverte" (open innovation) sur l'organisation des réseaux d'intégration des systèmes de la Défense. Ils raisonnent sur les stratégies de diffusion de connaissance et sur la gouvernance de la R\&D associées aux acteurs présents sur le segment des logiciels, un segment assez sensible puisque les technologies de l'information et de la communication sont désormais omniprésentes dans tous les systèmes et soussystèmes produits pour la Défense. Les stratégies analysées par les auteurs sont marquées par l'apparition de mécanismes hybrides, qui mêlent la référence à des logiciels propriétaires pour certains aspects des systèmes et à des modalités qui reposent sur les dynamiques spécifiques aux logiciels "libres". Dans le cadre de l'intégration de systèmes complexes, ces mécanismes prennent tout leur sens. Ils ne renvoient pas seulement à des questions de droits de propriété intellectuelle mais, aussi et surtout, à une question de stratégie de mobilisation des acteurs et à des questions de gouvernance de l'innovation. Dans ce nouveau modèle, l'acteur public perd alors une partie de sa compétence sur des pans entiers du système de Défense car un lien de dépendance est introduit envers les communautés propres à ces nouveaux acteurs qui apparaissent avec les logiciels "libres ", et qui ne sont pas mues par des logiques marchandes. La singularité des acteurs de la Défense (industriels, opérationnels ou institutionnels) repose sur leur démarche volontaire, qui est retenue pour trouver des solutions techniques indispensables à la gestion des systèmes de Défense, ou encore gérer des interfaces avec les legacy systems.

L'article de Thierry Kirat et Jean-Michel Oudot se concentre sur l'analyse économique des contrats d'armement, et propose une analyse des conditions d'exécution des marchés publics et de leur performance à travers les règles s'appliquant aux choix contractuels. Leur analyse porte sur la France, mais elle s'inspire largement des travaux qui ont été conduits par les auteurs en comparaison internationale avec les États-Unis et le Royaume-Uni. L'article détaille en particulier comment les modalités contractuelles impactent directement la coordination entre les acteurs de la Défense. Au-delà des règles formelles qui prévalent en matière de marchés publics, l'article montre aussi la prégnance des règles informelles à travers une investigation empirique de 48 contrats signés entre la DGA, agence d'acquisition du ministère français de la Défense, et 18 industriels français et européens entre 1994 et 2005. En empruntant à la fois à la théorie des coûts de transaction et à l'analyse économique du droit, les auteurs montrent soulignent les interdépendances entre les côtés offre et demande des contrats d'armement. 
Abel Ben Youssef et Gueorgui Ianakiev concentrent leur analyse sur les politiques industrielles fondées sur les offsets, logiques de compensation aux importations qui ne cessent de se généraliser dans les grands marchés d'Etat. Les taux moyens de compensation associés aux exportations américaines dépassent les $70 \%$, et dépassent très souvent les $100 \%$. Les auteurs caractérisent ce phénomène crucial pour la mise en place d'un marché européen des produits de Défense, et montrent combien il représente une somme de coûts d'information et de transaction. La présence d'externalités technologiques doit être prise en compte, et introduit des coûts d'opportunités indispensables pour appréhender la dynamique des compensations. La présence d'offsets limite de fait l'établissement de partenariats industriels supra-nationaux. Les auteurs indiquent que les effets de barrière se renforcent. Ils ne peuvent pas être supprimés simplement par l'introduction de nouvelles réglementations spécifiques, car c'est toute la philosophie des logiques de juste retour associées aux institutions européennes (OCCAr, AED) qui se trouve remise en cause.

Bernard Julien et Vincent Frigant développent une analyse sectorielle régionale fondée sur un travail empirique d'enquête assez rare, ancré dans la comparaison de l'usage des fonds de reconversion européens (Konver) dans plusieurs régions. En comparant l'Aquitaine, la Lombardie et la région de Manchester, ils montrent que les trajectoires productives sont essentiellement affectées par les niveaux nationaux, alors que les mesures initiées par les régions et les institutions européennes ont un impact marginal. L'efficacité des mesures n'est pas homogène, ce que les auteurs expliquent par la disparité entre les dispositifs locaux d'action publique. Au total, ces mécanismes montrent le rôle que jouent les trois dimensions réglementaire, normative et cognitive au niveau de la politique industrielle de reconversion. Les auteurs présentent une grille d'analyse qu'il serait intéressant de réutiliser dans d'autres contextes, par exemple pour tester d'autres mesures de politique industrielle au niveau régional.

\section{EN GUISE D'OUVERTURE...}

Ce numéro spécial espère convaincre que les politiques industrielles relatives à la Défense représentent un champ d'investigation pertinent pour les spécialistes des thèses institutionnalistes. Il livre les programmes d'armement et les politiques publiques de Défense avec des niveaux de granularité variés, qui couvrent à la fois des dispositifs de politique publique (les fonds de reconversion ou les offsets), des corpus réglementaires (les règles prévalant pour les marchés publics de Défense), l'évolution du rôle d'une agence de Défense particulière (la DGA en France) ou 
encore le management de certaines briques techniques (en matière spatiale ou de génie logiciel). Les organisations de Défense peuvent bénéficier de ces travaux, non seulement pour piloter les politiques publiques mais aussi pour mieux appréhender toutes les questions de gouvernance qui se rapportent à la préparation et à la mise en œuvre des systèmes de Défense. Cinq des six articles présentés dans ce numéro ont été directement mobilisés dans des réflexions menées par l'institution Défense dans la dernière décennie pour approfondir la connaissance de ses propres mécanismes et améliorer l'efficacité de ses processus. Ces articles permettent en retour de diffuser quelques éléments des recherches empiriques qui ont été conduites pendant ces projets.

Chavance (2007 : 103) concluait son introduction aux courants institutionnalistes significatifs par une section intitulée " objet limité, théorie générale". Les concepts mobilisés pour analyser ici les politiques industrielles de Défense contribueront à montrer avec ce numéro spécial la portée générale des théories institutionnalistes. Ces articles ne cherchent à faire système ni au niveau de la Défense, ni des politiques publiques, ni de la théorie économique, en particulier en gommant les singularités de chaque thèse institutionnaliste. En ce sens, les limitations de l'objet de la théorie dont parle Chavance sont celles que le chercheur emprunte aux grands auteurs de référence, Coase, North, Hodgson ou Williamson par exemple. Chaque article de ce numéro spécial privilégie des références et des nuances que le lecteur pourra comparer et apprécier. Avec ces références comme pour leurs prolongements possibles en matière de management de la connaissance ou d'économie de l'innovation, le système analytique n'est pas fermé. Au contraire. La complexité toujours croissante des relations entre l'État, le monde de la recherche et l'industrie justifie d'imaginer de nouveaux dispositifs d'action publique et des mécanismes de gouvernance adaptés. Les analyses présentées dans ces articles permettent d'imaginer de nouveaux moyens d'action, ciblant des coûts de transaction ou d'information, des référentiels de droits de propriété ou de coopération. Certains de ces dispositifs (par exemple la gouvernance des communautés) demeurent presque neutres au niveau budgétaire, alors qu'ils procurent des effets de levier importants et ouvrent la porte à un renouveau des politiques industrielles. Ces pistes méritent d'être fouillées, et les investigations liées à la nouvelle économie institutionnaliste poursuivies. 


\section{References}

Agassi, J., 1975, "Institutional individualism", British journal of sociology, volume 26, pp 144-155.

Amin, A., et Cohendet, P., 2004, Architectures of knowledge: firms, capabilities and communities, New York: Oxford university press.

Aoki, M., 2000, Information, corporate governance and institutional diversity, Oxford, New York : Oxford University Press.

Aoki, M., 2001, Towards a comparative institutional analysis, Cambridge (Mass): The MIT Press.

Bellais, R., \& Guichard, R., 2006, Defense innovation, technology transfers and public policy, Defence and Peace Economics, vol 17(3), pp 273-286.

Bishak, G, 1999, "Contending security doctrines and the military industrial base", in Markusen A. and Costingan K.K. eds; Arming the Future, a Defense industry for the 21st century, New York: Council of foreign relations book, pp 37-73.

Boland, L., 2003, The foundations of economic method, London : Routledge ( $2^{\text {nd }}$ edition)

Chavance, B., 2007, L'économie institutionnelle, Paris: La Découverte.

Coase, R., 2000, "L'économie néo-institutionelle ", Revue d'économie industrielle, volume 92 (1), pp 51-54.

Cohendet, P., et Lebeau, A, 1987, Choix stratégiques et grands programmes civils, Paris : Economica.

Coriat, B., et Weinstein, O., 2002, "Organisations, firms and institutions in the generation of innovation ", Research policy, vol 31, pp 273-290.

Coriat, B., et Weinstein, O., 2004, "Institutions, échanges et marchés ", Revue d'économie industrielle, ${ }^{\circ} 107$, 3ème trimestre.

Crozier, M. et Friedberg, E., 1977, L'acteur et le système, Paris : Seuil.

Dunne, J.P. (1995), "the Defense industrial base ", chapter 14 in Hartley \& Sandler eds ; 1995, pp 399-431.

Dussauge, P., et Cornu C., 1998, L'industrie française de l'armement, Paris : Economica.

Freedman, L., 1999, The politics of British Defence, 1979-1998, Macmillan Press.

Hartley, K., 1995, "Industrial policies and the Defence sector", chapter 16 in Hartley \& Sandler, eds; 1995, pp 459-490.

Hartley, K, \& Sandler, T., eds; 1995, Handbook of Defense economics, volume 1, Amsterdam: North Holland/Elsevier.

Headland, T. N., Pike K. L., et Harris M., eds; 1990, Emics and ethics: the insider/outsider debate, Sage.

Hobday, M., 1998. Product complexity, innovation and industrial organisation. Research Policy 26(6), 689-710.

27 Economie et Institutions $-\mathrm{n}^{\circ} 12 \& 13-2008-2009$ 
Hobday, M., Rush, H., Bessant, J., 2004. Approaching the innovation frontier in Korea: the transition phase to leadership. Research Policy 33(10), 1433-1457.

House of Commons Defence Committee, 2003, Defence procurement, Eighth report of session 2002-2003, reference HC694, London, July, 56pages.

Le Galès, P., 2003, Le retour des villes européennes. Sociétés urbaines, mondialisation, gouvernement et gouvernance, Paris : Presses de Science Po.

Lieberson, S., 1971, "The American study of military-industrial linkages ", The American Journal of Sociology, vol 74(4), pp. 562-584.

McGuire, M.C., 1995, "Defense economics and international security", Chapter 2 in Sandler \& Hartley, eds; 1995, pp 13-44.

Mérindol, V. \& Versailles D. W. (2007), International comparisons in the tryptich Government-Industry-Academic research in Defense-related R\&D projects: the crucial role of expertise abilities., chapitre 10 (137-56) in Sherif, M.H., et Khalil, M. (eds), Management of technology: New directions in technology management, Elsevier

Mérindol, V. \& Versailles D. W. (2010), Dual-use as knowledgeoriented policy : France during the 1990-2000s, International Journal of Technology Management, vol 50(2), pp

Mérindol, V., 2009, Gestion des compétences et gouvernance de l'innovation: la Défense dans l'économie fondée sur la connaissance. Paris: Economica (collection Recherche en gestion).

Mérindol, V., 2010, Défense et Stratégie: Penser autrement l'innovation Paris: Dalloz (collection Présage).

North, D., 1990, Institutions, institutional change and economic performance, Cambridge UK: Cambridge Univ. Press.

Pavitt, K., 2003, Specialization and systems integration, chapter 5 (78-91) in Prencipe Prencipe Andrea, Davies Andrew, Hobday Michael (eds), The Business of Systems Integration, New York: Oxford University Press.

Prencipe, A., Davies, A. \& Hobday, M., (Eds;). (2003). The business of systems integration. Oxford: Oxford university press.

Sandler, T. et Hartley, K., 1995, The Economics of Defense, Cambridge (UK): Cambridge University Press.

Scharpf, F.W., 1997, Games real actors play: Actor-centred institutionalism in policy research, Westwiew press.

Schmidt, C., 1987, "Peace and war economics in retrospect: some reflections on the historical background of Defence economics", chapter 2 in Schmidt \& Blackaby, eds; 1987, Peace, Defense and economic analysis, London: Macmillan Press. 
The British Ministry of Defence, 2005, Defence industrial strategy, presented to the Parliament by the Secretary of State for Defence, December, London.

The British Ministry of Defence, 2006, Defence technology strategy for the demands of the $21^{\text {st }}$ century, London, 196pages.

Versailles, D. W., Mérindol, V., Cardot, P., 2003, Recherche et technologie, enjeux de puissance, Paris: Economica

Versailles, D. W., ed ; "Réseaux de connaissances, réseaux de Défense ", numéro thématique de la Revue d'économie industrielle, $\mathrm{n}^{\circ} 112 ;$ 4ème trimestre.

von Hippel, E., 1986. "Lead users: a source of nouvel product concepts". Management Science 32(7), 791-805. 